\title{
Multitud libre y producción del deseo común en Spinoza
}

\section{Spinoza's Free Multitude and the Production of Common Desire}

\author{
Aurelio Sainz Pezonaga ${ }^{1}$ \\ Universidad de Castilla-La Mancha (España)
}

Recibido: 23-06-18

Aprobado: 18-09-18

\section{Resumen}

El texto considera las dos dicotomías del deseo productor de juicios de valor en Spinoza: deseo pasional versus deseo racional y deseo ético versus deseo político, y explica por qué todo acuerdo sobre valores posee un carácter político. Atiende, luego, al modo en que construye lo que llama "dictámenes de la razón" a través de la idea universal de hombre y la noción de mal. Revisa la socialidad tejida espontáneamente por la imitación de los afectos y la cooperación intelectual de la multitud. Y, por último, esboza el concepto de deseo común adecuado a la multitud libre. Su tesis es que el deseo común de concordia que mueve a la multitud libre se constituye, paradójicamente, en la disputa por las definiciones compartidas de lo bueno y lo malo, esto es, en la disputa en torno a la institución del imperium.

Palabras-clave: Spinoza, multitud libre, deseo común, conflicto, concordia, Estado.

\footnotetext{
${ }^{1}$ (Aurelio.Sainz@uclm.es) Profesor asociado de Filosofía en la Universidad de Castilla-La Mancha y profesor de enseñanza secundaria, es autor de Contra la ética, por una ideología de la igualdad social (Madrid, Debate, 2002) y de Rupturas situacionistas. Superación del arte y revolución cultural (Ciempozuelos, Tierradenadie, 2011). Tradujo a Warren Montag, Cuerpos, masas, poder. Spinoza y sus contemporáneos (Ciempozuelos, Tierradenadie, 2005) y editó Escritos sobre el arte de Louis Althusser et al. (Ciempozuelos, Tierradenadie, 2011). Entre otros autores y asuntos, ha escrito artículos sobre Spinoza como "El deseo activo de amar (afecto y materialismo en Spinoza)", (Youkali. no 3, mayo 2007), "Universalismo y singularidad en la Ética de Spinoza", (en Montserrat Galcerán Huguet y Mario Espinoza Pino (eds.), Spinoza contemporáneo, Ciempozuelos, Tierradenadie, 2008) o "Spinoza y la pregunta por la solidaridad” (en Luis Alegre y Eduardo Maura (eds.), ¿Qué es la ilustración?, Madrid, Guillermo Escolar Editor, 2017). Pertenece a los consejos editoriales de las revistas en línea Youkali, Décalages, Demarcaciones y Círculo Spinoziano, así como al de Tierradenadie ediciones y al de la colección Spinoziana de Mimesis Edizioni. Es secretario del Seminario Spinoza. El presente artículo forma parte de la investigación encaminada a la obtención del titulo de Doctor, dirigida por Francisco Javier Espinosa Antón, a quien agradezco su enseñanza y estímulo.
} 


\begin{abstract}
The text considers Spinoza's two dichotomies of desire as producer of value judgments: passionate desire versus rational desire and ethical desire versus political desire, and explains why any agreement on values has a political character. It then deals with the way in which he constructs what he calls "dictates of reason" through the universal notion of man and the idea of evil. It looks at the sociality spontaneously woven by the imitation of the affects and the intellectual cooperation of the multitude. And, finally, it outlines the concept of common desire suited to a free multitude. Its thesis is that the common desire for harmony that moves a free multitude to act is constituted, paradoxically, in the dispute for the shared definitions of good and evil, i.e., in the dispute over the institution of imperium.
\end{abstract}

Key-words: Spinoza, free multitude, common desire, conflict, harmony, State.

En la filosofía política de Spinoza, un juicio de valor compartido sólo puede ser producido por un deseo compartido, un juicio de valor común, por un deseo común. Al menos eso es lo que se desprende de su concepción del deseo como causa de los valores. En esta línea, la sociedad se define como un proceso de constitución de deseos comunes, proceso, también, de lucha por constituirlos de un modo o de otro. Cuando Spinoza habla, pues, del mejor imperium $^{2}$, cabe entender que se refiere a aquel proceso social a través del cual la multitud libre configura un deseo común más potente. El mejor imperium será el deseado con una mayor intensidad por la multitud, el que despliegue las condiciones para la formación del deseo común de mayor potencia: un deseo productor de amor por la concordia. El mejor imperium es el que la multitud libre instituye cuando se adentra en un ciclo de liberación política, que, a su vez, alimenta, y se retroalimenta, de un ciclo de liberación ética. Y es "mejor" no por la autoridad de Spinoza, sino en cuanto lo afirme el deseo de la multitud libre que como tal lo inviste al instituirlo.

\footnotetext{
${ }^{2}$ Al igual que Bernard Pautrat en su versión francesa del Tratado político de Spinoza, y por sus mismas razones, mantenemos el término "imperium" sin traducir a lo largo del artículo. A imperium le ocurre como a conatus. Es un concepto claramente definido por Spinoza -el derecho que se define por la potencia de la multitud (TP 2/17)-que pierde nitidez al intentar buscar un término equivalente en español. Cfr. Bernard Pautrat, "Pourquoi retraduire le Tractatus politicus?”, [en Baruch Spinoza, Traité politique, París, Allia, 2013].
} 


\section{El conatus, causa de los juicios valorativos}

Spinoza reconoce una única fuente de los juicios de bueno y malo: nuestro conatus, nuestro apetito o deseo. "Nosotros -dice- no intentamos, queremos, apetecemos ni deseamos algo porque lo juzguemos bueno, sino que, al contrario, juzgamos que algo es bueno porque lo intentamos, queremos, apetecemos y deseamos" (E 3P9S). El conatus es la fuente de los juicios de valor en el sentido de que es su causa eficiente. Es, entiéndase, una causa eficiente que el finalismo considera como causa primera debido a que ignora su realidad de causa causada y de expresión de la potencia divina. El conatus es, por tanto, la causa eficiente de cualquier fin u objetivo que nos propongamos a nivel particular o que proyectemos a nivel colectivo ${ }^{3}$.

El conatus, el apetito o el deseo como causas de los valores se modulan de dos maneras distintas: como deseos pasionales y como deseos racionales, según que el conatus sea causa parcial o causa adecuada del juicio. Y, a su vez, estas dos modulaciones poseen una vertiente o un ángulo ético y una vertiente o ángulo político, dependiendo de si conciernen a la potencia de un individuo humano o a la de una multitud.

Spinoza define el bien y el mal pasionales y su diversidad y conflictividad en E 3P39S. Sobre el deseo racional, se explica expresamente en las proposiciones 58 y 59 de la Parte III de la Ética. En esta última, Spinoza remite a la fortitudo todos los afectos que se refieren a la mente en tanto que entiende. Y, dentro de ella, distingue entre animositas y generositas, según se trate de un deseo racional de conservar el ser o de "ayudar a los demás hombres y unirse a ellos en amistad", respectivamente. Un deseo racional es, según este escolio, "un deseo por el que cada uno se esfuerza en virtud del solo dictamen de la razón".

Las consecuencias para la producción de valores desde el deseo racional las expone Spinoza en E 4P26 y E 4P27. Si juzgamos algo como bueno porque lo deseamos, aquello que deseemos en cuanto obramos, a saber, en cuanto concebimos ideas adecuadas, lo juzgaremos bueno y nuestro juicio será verdadero. Lo verdaderamente bueno o verdaderamente útil es lo que deseamos racionalmente; y esto no es otra cosa que el conocimiento mismo y lo que sirve al conocimiento.

Al deseo pasional político le dedica Spinoza el primer artículo del capítulo VI del Tratado político.

Dado que los hombres se conducen, como hemos dicho, más por el afecto que por la razón, la multitud conviene de manera natural (naturaliter convenire), y quiere conducirse como una sola mente ${ }^{4}$, no porque la mueva la razón, sino

\footnotetext{
3 Cfr. E 4P.

4 Sobre esta traducción de "una veluti mente duci", cfr. Francisco Javier Espinosa, "Être une multitude et agir comme une seule âme", [en Maria Luísa Ribeiro Ferreira, Diogo Pires Aurélio, 
algún afecto común, es decir (como dijimos en el $§ 9$ del capítulo III), por una esperanza o un miedo comunes o por el anhelo de vengar una injuria común. Por otra parte, el miedo a la soledad habita en todos los hombres, puesto que nadie, en solitario, tiene fuerzas para defenderse ni para procurarse los medios necesarios de vida. De ahí que los hombres apetezcan por naturaleza (natura appetere) el estado civil, y es imposible que ellos lo destruyan jamás del todo.

De este artículo se deben señalar al menos tres cuestiones. La primera es que el pasaje indica que las mismas causas que llevan a la constitución de la sociedad, impulsan las conspiraciones contra los regímenes ya existentes, tal como apunta la remisión a TP 3/9. En un reconocido artículo, "L' indignation et le conatus de l'État spinoziste" (1994), Alexander Matheron analiza ambos pasajes enfatizando el papel que desempeña la indignación como generadora de rebeliones y como creadora de sociedades ${ }^{5}$.

La segunda es que el parágrafo establece de manera directa la relacionalidad ${ }^{6}$ como base de cualquier comunidad política ${ }^{7}$. Apela para ello al carácter natural de la multitudo y del status civilis y al miedo universal a la soledad. La socialidad es intrínseca al conatus, incluso en sus efectos pasionales.

Por último, las pasiones aparecen en TP $6 / 1$ ya no como causa de conflicto como en TP $1 / 5$, sino como causa de la conveniencia, del convenire natural de la multitud y de la creación del estado civil.

La capacidad productiva y reproductiva de lo social que posee la indignación reside en que, a pesar de ser una forma de odio, se sostiene al mismo tiempo en la imitación de los afectos, esto es, en la identificación o en el amor a la víctima, que son formas de alegría y facilitan la reciprocidad. El problema es pensar cómo es posible que el miedo o el anhelo de venganza, en tanto que pasiones tristes, puedan ser comunes, puedan unir, esto es, supongan una conveniencia de naturaleza entre distintos seres humanos, ya que convenir en naturaleza (natura convenire) significa "convenir en potencia y no en impotencia o negación (negatione)" (E 4P32).

Oliver Feron (eds.), Spinoza, ser e agir, Lisboa, CFUL, 2011 ], p. 146.

${ }^{5}$ Cfr. Alexander Matheron, "L'indignation et le conatus de l'État spinoziste", [en Études sur Spinoza et les philosophies de l'âge classique, op. cit.], p. 225 y ss.

${ }^{6}$ En la ontología de Spinoza, la relacionalidad es una característica propia de todos los modos finitos como tales modos finitos, incluido cada uno de los seres humanos. Es el hecho de que para los modos finitos su relación con otros modos, sean unos u otros, pero siempre en plural y en, al menos, dos niveles de integración, es constitutiva, no accidental. El texto clave sobre la concepción conjunta del conatus y la relacionalidad (que es siempre múltiple) en la filosofía de Spinoza es Étienne Balibar, "Individualité et transindividualité chez Spinoza", [en Spinoza politique. Le transindividuel, París, PUF, 2018], pp. 199-244; en concreto las pp. 220-221. Cfr. también la lectura del concepto de forma en Spinoza que realiza François Zouravichbili en Spinoza. Une physique de la pensée, París, PUF, 2002, p. 73 y los dos primeros capítulos de Vittorio Morfino, El tiempo de la multitud, Ciempozuelos, Tierradenadie ediciones, 2013.

${ }^{7}$ Como explicita la última frase y subraya el artículo siguiente, TP 6/2. 
Siendo la tristeza un afecto por el que se disminuye o reprime la potencia de obrar del cuerpo (E 4P41) y no pudiéndose atribuir a la mente en cuanto que actúa (E 3P59), no puede conducir por sí misma a una conveniencia o mutuo favor entre seres humanos. Sólo la alegría de los demás, que nunca es directamente mala (E 4P41), puede ser común, puede unirnos a otras personas, puede convertirse en algo útil para mí así como la mía lo será para ellos (E 4P31C). Si el odio o el miedo sirven para unir a las personas, no es por la disminución de potencia que ellos suponen, sino por la alegría que nos produce imaginar que se destruye aquello que odiamos o tememos (E 3P20).

La aversión o el miedo no pueden unir por sí mismos. Lo que nos junta es el esfuerzo por alejar la causa del daño o bien la alegría de imaginar la exclusión de lo que nos produce tristeza. En la medida en que el odio o el miedo son una impotencia, una tristeza, no se pueden compartir, porque nada positivo hay en ellos, son sólo la disminución de nuestra fuerza vital. El miedo universal a la soledad, que sería la pasión más fuerte de las citadas en TP 6/1, no puede unir como tal miedo, sino por el lado de la esperanza que necesariamente incluye (E 3DA13E). La otra cara del miedo común de soledad es la esperanza común de concordia o el apetito de vida social, que ya es un germen de concordia.

Traducida esta última cuestión al problema de la creación de los valores de bueno o malo, resulta que las pasiones pueden generar tanto una diversidad irreconciliable de juicios valorativos como los valores compartidos socialmente, los valores comunes. De hecho, esto es lo específico de la política racional que, reconociendo la irreductibilidad del conflicto, trabaja para contrarrestar unas pasiones con otras, unos poderes con otros ${ }^{8}$. Los valores comunes son ocasionados por deseos comunes, que en la medida en que existen y se fortalecen, ponen freno al conflicto pasional.

\section{No hay acuerdo posible sobre lo que está bien o mal que no sea político}

Tanto el conflicto como la conveniencia pasional remiten a la esencial relacionalidad múltiple del ser humano como modo de la sustancia, esto es, implican un esfuerzo por perseverar en el ser de unos seres singulares entre seres singulares. Y, no obstante, se inscriben en posiciones contrarias. La conveniencia pasional duradera institucionaliza una lucha contra el conflicto pasional. La sociedad perdura en la medida en que neutraliza el conflicto entre la diversidad irreconciliable de valoraciones y lo hace instituyendo un sistema valorativo común': un derecho común, un imperium.

\footnotetext{
${ }^{8}$ Cfr. TP 1/6 y TP 6/3. Aquí rige E 4P1 y su escolio.

9 Sobre la neutralización política del conflicto pasional y la institución de la valoración en Hobbes, cfr. Leviatán, XIII, 13 y Leviatán, XV, 36.
} 
De cuanto hemos explicado en este capítulo resulta claro que en el estado natural no existe pecado o que, si alguien peca, es contra sí y no contra otro. Por derecho natural nadie, en efecto, está obligado, si no quiere, a complacer a otro ni a considerar bueno o malo sino aquello que, según su criterio personal, juzga como tal. En una palabra, por derecho natural nada es prohibido, excepto lo que nadie puede realizar (ver el $\S 5$ de este capítulo). En cambio, el pecado es una acción que no puede ser realizada según derecho... El pecado no se puede concebir, pues, más que en el imperium, esto es, allí donde lo que es bueno y lo que es malo se determina a partir del derecho común del imperium entero, y donde nadie actúa con derecho (por el $\S 16$ de este capítulo), sino en cuanto actúa en virtud de una decisión común o consenso. Pues (como dijimos en el artículo anterior) es pecado lo que no puede hacerse o está prohibido por el derecho, mientras que obediencia es la voluntad constante de ejecutar lo que es bueno según derecho y que, por decisión común, debe ser puesto en práctica (TP 2/18-19).

Este pasaje del capítulo II del Tratado político es casi idéntico al párrafo final del escolio 2 de la proposición 37 de la Parte IV de la Ética, donde quizás se expone el problema de una manera algo más directa: "en el estado natural no hay nada que sea bueno o malo en virtud del consenso de todos" ${ }^{\prime 10}$. Lo que está en juego es qué valoración puede alcanzar una aceptación efectiva de todos, qué valoración puede tener una cierta objetividad, lo que significa, a su vez, una cierta materialidad, una cierta realidad efectiva. La tesis de Spinoza es que eso sólo puede ocurrir en el estado civil, a saber, en el momento en el que la multitud instituye un imperium y una civitas que sujetan esa valoración con amenazas y promesas. Lo malo es lo prohibido y Spinoza lo llama pecado o desobediencia. Y lo bueno es lo permitido y es ejercido por la obediencia. No hay otra forma de consensu efectivo que la que pasa por la civitas.

Las consecuencias para la ética de este posicionamiento son de enorme calado. No hay acuerdo posible sobre lo que está bien o mal que no sea político. Y será político o porque lo establece el derecho común o porque resulta de una conspiratio. El deseo racional como causa de valoración se sitúa entonces en esa dicotomía. O bien contribuye a la fundamentación de una estructura social o bien conspira para rehacerla. En ese filo político se encuentra toda filosofía, pero muy en concreto la filosofía de Spinoza. La filosofía o bien sirve al imperium o bien conspira contra él en la teoría. Cuando Spinoza enuncia en esta proposición 37 de la Parte IV de la Ética que "El bien que apetece para sí todo el que persigue la virtud, lo deseará también para los demás hombres, y tanto más cuanto mayor conocimiento tenga de Dios", está haciendo una declaración política. Está planteando que buscar la virtud para uno mismo implica buscar compartirla, tenerla en común con los demás, alcanzar un consenso común sobre ella, pero esto no puede suceder, no puede lograrse,

\footnotetext{
${ }^{10}$ Cfr. también los capítulos XVI y XIX del Tratado teológico-político.
} 
si no se dan las condiciones políticas adecuadas. Es más, la extensión de la virtud es una acción política por sí misma, porque busca convertirse en civitas. Claro que la única manera en que Spinoza puede defender racionalmente esa extensión de la virtud es a través de la libre comunicación. Así, la libertad de expresión se ha de entender desde esta óptica como una forma de regular la conspiración teórica, de convertirla en un derecho común. Mientras que la democracia regula la posibilidad de cambiar de valoraciones compartidas efectivas y, de esa manera, institucionaliza el derecho común a la conspiración, entendida esta en un sentido completo, teórico y práctico.

\section{Los dictámenes de la razón}

Spinoza dedica toda la Parte IV de la Ética a desarrollar una idea del deseo racional como causa de las nociones de bien y mal. Como explica en el Prólogo, a pesar de que las nociones de bien y mal son modos de pensar o ficciones que no indican nada positivo en las cosas, sino sólo el deseo de la persona, es posible producirlas también racionalmente, es decir, pueden resultar del deseo de "formar (formare) una idea de hombre como modelo (exemplar) de naturaleza humana que tener a la vista". Este deseo se integra, como dice al principio del Prólogo, en la voluntad de conocer la causa de nuestra servidumbre y la diferencia de unos afectos y otros respecto a nuestro esfuerzo por perseverar en el ser, y es, por ende, un deseo racional.

La desviación racional de una imaginación como es la idea universal de hombre para formar un modelo de naturaleza humana permite convertir las nociones comunes - principalmente, el conatus y la relacionalidad múltiple- en esencias, y las esencias en ideales normativos o modelos. De esta forma, el bien y el mal se definen como si el conatus y la relacionalidad no fueran nociones compartidas por todos los modos de la sustancia, sino propias exclusivamente de una imaginaria esencia humana ${ }^{11}$. Actuando así se procede de la misma manera en que, habitualmente, lo hace la práctica finalista, esto es, aquella que conoce la acción, pero no sus causas. Con la diferencia de que, ahora, ese proceder, esa

${ }^{11}$ E 2P37. Chantal Jacquet habla a este respecto de un "relativismo verdadero", que no deja de ser una forma paradójica de plantear la cuestión. Cfr. Chantal Jaquet, Les expressions de la puissance d'agir chez Spinoza, París, Éditions de la Sorbone, 2005, p. 88. Cfr. también André Tosel, "Poïétique et modèle de l'accomplissement humain", [en André Tosel, Spinoza ou l'autre (in)finitude, París, L'Harmattan, 2008], pp. 211-236, donde Tosel intenta resolver el problema del modelo de naturaleza humana sin pasar por la imaginación, a mi juicio, sin éxito. Alexander Matheron, por su parte, plantea la necesidad de distinguir entre la norma estratégica (opératoire), que construye Spinoza, y la norma ontológica, que rechaza como ilusión finalista. Cfr. Alexander Matheron, "État et moralité selon Spinoza", [en Études sur Spinoza et les philosophies de l'âge classique, op. cit.], p. 187. Para una ampliación de mi punto de vista sobre la idea de hombre spinoziana, cfr. Aurelio Sainz Pezonaga, "Universalismo y singularidad en la Ética de Spinoza", [en Montserrat Galcerán Huguet y Mario Espinoza Pino, Spinoza contemporáneo, Ciempozuelos, Tierradenadie ediciones, 2009], pp. 133-160. 
construcción de modelos, se convierte en un imaginationis auxilium, de modo similar a la medida, el tiempo y el número de la carta XII a Meyer (Ep 12: 57-59), esto es, en un recurso imaginativo al servicio del conocimiento. La mente puede utilizar la potencia de la imaginación en la medida en que la libera, es decir, si al tiempo que imagina cosas inexistentes, sabe que no existen (E 2P17S).

Al conatus y la múltiple relacionalidad en tanto que principios normativos, los llama Spinoza "rationes dictamina" (E 4P18S) o "leges humanae rationis" (TP 2/8) y de las personas que hacen suyos estos principios afirma que actúan "ex ductu rationis" (E 4P24). La razón, en estas expresiones, no es otra cosa que el conocimiento de nociones comunes (E 2P40S2), principalmente, como lo demuestra E 4P18S y la distribución de los capítulos del Apéndice de la Parte IV, del conatus y la múltiple relacionalidad. Una de esas nociones comunes es el conocimiento de la potencia del conocimiento, del conatus de la mente, esto es, el carácter reflexivo de la verdad, índice de sí misma y de lo falso (E 2P43 y su escolio).

La reflexividad de la idea adecuada implica, además, un amor al conocimiento verdadero. Vivir según los dictámenes de la razón o conducido por ella significa conocer lo que aumenta nuestra potencia, esto es, lo que nos ayuda en nuestro esfuerzo por perseverar en el ser, y amar ese conocimiento. $\mathrm{Y}$ aquello de lo que podemos tener certeza de que aumenta nuestra potencia es el conocimiento de que nuestra esencia es el deseo de vivir y nuestra vida, una múltiple relacionalidad. Enmarcar estos conocimientos en una idea universal de hombre y convertir el resultado en un espejo (exemplar) de naturaleza humana no significa, sin embargo, que esos ideales existan de alguna otra forma que como imaginaciones.

\section{La noción de mal}

Los dictámenes de la razón son aquello que las ideas adecuadas afirman o niegan y, en primer lugar, la afirmación reflexiva de la idea adecuada como verdadera y como causa de alegría, esto es, buena. Ahora bien, si esto es así, ¿por qué es necesario realizar la desviación por el universal imaginario? ¿No sería suficiente con extraer las consecuencia de ese conocimiento, como parece bastar en la Parte V de la Ética?

El problema es que hay algo que las ideas adecuadas no nos ofrecen ni nos pueden ofrecer; y ese algo es la noción de mal. "El conocimiento del mal es un conocimiento inadecuado", afirma Spinoza en E 4P64, ya que el conocimiento del mal no es otra cosa que la conciencia de la tristeza, que depende de ideas inadecuadas. Y añade en el corolario: "si la mente humana no tuviera más que ideas adecuadas, no formaría ninguna noción del mal”. 
La única manera de elaborar la idea imaginaria de privación es a través del universal imaginario. Esta sería la enseñanza que Spinoza extrae del intercambio epistolar con Blijenbergh. En la media en que no podemos escapar completamente de las pasiones y, en concreto, de las pasiones tristes, como el odio, ya que somos una parte de la naturaleza (E 4P4), pero deseamos perfeccionar nuestro entendimiento (E 4C4), no podemos dejar de formar universales imaginarios que nos permitan entender ese rechazo a partir del cual juzgamos algo como malo.

Un pasaje de la carta XIX a Blijembergh expone el procedimiento por el que se construye la idea de mal.

El mal que en ella [la acción de Adán] había no era otra cosa sino la privación de un estado más perfecto, que, por efecto de su acción, Adán debía perder. Es algo claro que la privación no es nada positivo y que solo se llama así en virtud de nuestro entendimiento pero no del divino. Esta noción de privación nace de que expresamos todas las cosas singulares del mismo género, por ejemplo, todas las que tienen figura externa humana (externam hominum figuram), mediante una definición única e idéntica para todas, y juzgamos que todas ellas son igualmente capaces de la más alta perfección deducible de tal definición. Ocurre así que cuando encontramos un individuo cuyo obrar no se corresponde con esta perfección, lo juzgamos privado de ella y desviado de su naturaleza; lo cual no haríamos si no lo hubiéramos sometido previamente a aquella definición y no le hubiéramos asignado una naturaleza correspondiente (Ep 19: 91).

Juzgamos algo malo porque lo repudiamos, lo odiamos porque nos produce tristeza (E 3P13S) y nos produce tristeza porque le hemos asignado una naturaleza, una perfección, a las que nos apegamos afectivamente, y lo imaginamos privado o desviado de ellas. La tristeza resulta de la frustración que para nosotros supone imaginar algo privado de la naturaleza que nosotros desearíamos que tuviera. Esto ocurre, por ejemplo, cuando nos hemos formado un modelo de casa al que hemos ligado una preferencia y vemos una vivienda que no se ajusta al modelo. Esa discrepancia es causa de tristeza porque se opone al modelo de casa que amamos (E 3P19) y nos lleva a juzgar la construcción como imperfecta (E 4P: 162).

\section{La imitación de los afectos}

Un proceder similar se despliega con la valoración de las cosas no producidas por el ser humano (E 4P). Pero si hablamos de mal en términos de las acciones humanas, el proceso es algo más complejo, ya que interviene el fenómeno que Spinoza denomina "imitación de los afectos (affectuum imitatio)": "Si imaginamos que una cosa semejante a nosotros, sobre la cual 
no hemos proyectado afecto alguno, experimenta algún afecto, nosotros experimentamos un afecto semejante" (E 3P27).

Esto es, a diferencia del modelo de casa o de caballo, sobre los que tenemos que crear una ligazón afectiva para producir una valoración, con nuestros semejantes el vínculo afectivo es inmediato. La semejanza imaginada se expresa en afecto semejante. Pero esto ocurre en un sentido doble y de modo recíproco con otras personas. Para nosotros, en efecto, la semejanza imaginada es la idea universal de hombre formada, en su funcionamiento más básico, a partir de la figura humana ${ }^{12}$, como decía Spinoza en la carta XIX a Blijembergh, y que puede modularse de muy diversas maneras (E 2P40S1).

Pero, el caso es que no sólo imitamos los afectos que imaginamos de nuestros imaginados semejantes, sino también los deseos que imaginamos de ellos (E 3P27S). La emulación, el contagio de los deseos, vuelta sobre nosotros mismos, significará, a su vez, que deseemos imaginar que nuestros imaginados semejantes nos deseen (E 3P29).

La idea universal de hombre funciona a un doble nivel imaginario. Está por un lado el hecho de que está formada imaginariamente como cualquier idea universal tal como explica E 2P40S1. Por el otro, a partir de ese primer nivel, la imaginación produce deseos y, por tanto, valoraciones. La imitación provoca el deseo de agradar a nuestros semejantes imaginados y el deseo de que nos agraden. Se genera así la alabanza y el vituperio de unos a otros (E 3P29S). Ahora, la idea universal funciona como operador del carácter social de los afectos, como herramienta del mutuo control de las acciones o dispositivo de (re)producción social ${ }^{13}$.

Y no sólo eso, sino que dirige igualmente la interiorización de la regulación social, esto es, determina el modo en que consideramos nuestra acciones desde un punto de vista externo (E 3P30). La gloria y la vergüenza, el contento de sí mismo y el arrepentimiento son el resultado del mecanismo de regulación social en el que deviene la idea universal de hombre (E 3P30S).

El deseo racional mantiene, por tanto, la idea universal de ser humano porque, siendo como somos una parte de la naturaleza, no puede dejar de pensar la privación e integrarla en el conocimiento. Pero, sabemos que no hay una sola idea universal de hombre, sino tantas como experiencias y cerebros (E 2P40S1). Sabemos, además, que son fuente de conflicto (E 3P31S).

${ }^{12}$ La inmediatez del vínculo afectivo que posee la figura exterior humana y su disponibilidad básica a la hora de dar contenido imaginario a la idea universal de ser humano comunica, en la distancia de los siglos, con la importancia del rostro para el "antispinozista" Emmanuel Lévinas y con la fase del espejo de Jacques Lacan.

13 Tanto Alexander Matheron (Individu et communauté chez Spinoza, París, Les Éditions de Minuit, 1988, pp. 154-179 y 320-327; y “L'indignation et le conatus de l'État spinoziste", [en Études sur Spinoza et les philosophies de l'âge classique, op. cit.], p. 225 y ss.), según hemos ya anotado, como Étienne Balibar (Spinoza et la politique, op. cit., pp. 101 y ss.) han postulado y argumentado este papel fundamental de la imitación de los afectos en la génesis o producción y reproducción de la sociedad. 
Lo mismo cabe entender de la privación, del mal. La definición imaginaria del mal, que se deriva de la definición de la naturaleza humana, es parte efectiva de lo social y objeto crucial de disputa. Definir el mal conlleva determinar lo que debe ser evitado, excluido, reprimido o destruido. La ética, entonces, no puede dejar de intervenir en la discusión, la razón no puede abandonar la disputa en torno a la definición del mal por muy imaginaria que sea. En ella se pone en juego, con consecuencias decisivas, la forma de vida que vamos a desplegar individual y colectivamente.

La efectividad y el carácter polémico de la definición del mal son signo de la servidumbre humana. Pero también demuestran que la ética no puede prescindir de la política, que para conocer la mente y su felicidad no podemos olvidarnos de su relacionalidad, de su socialidad. La paradoja del hombre libre, el que el hombre nacido libre no formaría ningún concepto del bien y del mal (E 4P68), esto es, no formaría ninguna noción universal de hombre libre, es la prueba del carácter desviado de ésta, del hecho de que la idea universal es un rodeo constructivo en el proceso de perfeccionamiento de la mente que desemboca en el amor intelectual de Dios (E 4C4). La libertad humana que se aborda en la Parte V de la Ética no requiere atender a la privación, no precisa valoraciones morales, el conocimiento y la alegría que brota de su práctica se alimentan circularmente ${ }^{14}$.

\section{La cooperación intelectual de la multitud}

Veamos, entonces, cómo esta normatividad racionalmente construida de la Parte IV de la Ética es recogida en el Tratado político. TP 2/8 es el antecedente filosófico, dentro de la obra, de la distinción entre gobernar con derecho y gobernar bien que abre el capítulo V. Contiene, además, un fragmento que Spinoza copia literalmente del TTP, lo que permite pensar que expone una tesis fundamental de su investigación: "aquello que la razón dice que es malo, no es tal respecto al orden y a las leyes de la naturaleza universal, sino tan sólo respecto a las leyes de la nuestra".

Spinoza defiende que, desde el punto de vista de la naturaleza universal, todo vale. Todas las acciones, más allá del juicio que podamos producir sobre ellas, tienen la misma validez, la misma legitimidad, el mismo derecho a existir y perdurar. Sin embargo, todas las acciones no generan los mismos efectos, no

\footnotetext{
${ }^{14}$ Cfr. Chantal Jacquet, Les expressions de la puissance d'agir chez Spinoza, op. cit., p. 89. Tanto la carta XIX a Blyembergh como el cap. IV del TTP insisten en esta idea de "obrar bien y buscar el bien en tanto que bien y no en tanto que contrario al mal, es decir, de buscar el bien por amor del bien, no por temor del mal" (TTP 4: 66) que remite a E 4P63C como transición hacia la Parte V de la Ética. En la distinción entre la multitud libre y la multitud sierva, esta característica se convierte en la oposición entre cultivar la vida y evitar la muerte.
} 
nos pueden por tanto dar igual unas u otras, los efectos de unas aumentan nuestra potencia, los de otras la disminuyen. Spinoza separa legitimidad y valoración. Las valoraciones éticas o políticas no están sometidas a la legitimidad. El bien no procede ni se define por la validez, sino que sigue a la potencia.

En el contexto de la filosofía política del siglo XVII, la legitimidad política, entendida como el derecho a mandar y, por tanto, a ser obedecido, depende o bien del derecho natural divino o bien del derecho natural subjetivo. La herencia de Tomás de Aquino para el primer caso. Thomas Hobbes como figura destacada del segundo.

En el primer caso, si el gobernante y el régimen político se adecúan al derecho natural establecido por Dios, eso los convierte en un buen gobernante y un buen régimen. El gobernante es bueno porque se somete a la ley natural, parte de la ley eterna de Dios. El régimen es bueno porque cumple el fin al que ha sido destinado por Dios. El monarca, pues la monarquía es el régimen más perfecto, sigue los mandatos de un Dios a quien, en última instancia, debe su poder y a quien imita en su reino. Por el mismo derecho natural, si el rey es buen cristiano, si procura la vida buena de la multitud orientada hacia su felicidad celestial, sus súbditos tienen la obligación religiosa de obedecerle. La desobediencia al buen gobernante equivale a la desobediencia a Dios y es, por tanto, pecado mortal. Y si sus mandatos van contra Dios, los súbditos no tienen la obligación de obedecerle ${ }^{15}$.

En el segundo caso, el derecho natural subjetivo descansa en el origen contractual del poder político. El mantenimiento de la promesa supuestamente comprometida en el pacto que acaba con la guerra de todos contra todos es lo que obliga a los gobernados a obedecer. Un gobierno actúa con derecho cuando ejerce su poder dentro del marco establecido por el pacto, la instauración de la paz, y es entonces legítimo, lo que significa que los súbditos tienen también el imperativo racional de obedecerlo, de cumplir su promesa originaria.

La valoración de buen o mal gobierno deriva en las teorías iusnaturalistas, teológicas o contractualistas, de la legitimidad, es decir, del derecho o falta de derecho de los gobernantes a dirigir la sociedad ${ }^{16}$.

La tesis spinoziana de la equivalencia entre derecho y potencia -"tantum juris quantum potentia"- elimina, sin embargo, la posibilidad de realizar semejante derivación, lo cual muestra su distancia ya en el TTP respecto a la tradición contractualista. Para Spinoza, todos los gobiernos, en la medida en que ejercen el poder político, tienen derecho a hacerlo. Sin embargo, eso no significa que lo hagan bien $^{17}$, porque la bondad o maldad no va ligada a tener o

\footnotetext{
${ }^{15}$ Cfr. Tomás de Aquino, La monarquía, Madrid, Tecnos, 1989 y Tomás de Aquino, Suma de Teología IV, Madrid, BAC, 1994, II, II, 104-105, pp. 211-222.

${ }^{16}$ Cfr. Francisco Colom González, "Legitimidad política" [en Fernando Quesada (ed.), Filosofia política I. Ideas políticas y movimientos sociales, Madrid, Trotta, 2002], pp. 171-186.

${ }^{17}$ Esta sería la diferencia con Hobbes, para Hobbes tienen derecho y, por eso, lo hacen bien, aunque siempre puede hacerse mejor o peor.
}

Araucaria. Revista Iberoamericana de Filosofia, Política, Humanidades y Relaciones Internacionales, año $21, \mathrm{n}^{\circ} 42$. Segundo semestre de 2019. Pp. 23-44. ISSN 1575-6823 e-ISSN 2340-2199 doi: 10.12795/araucaria.2019.i42.02 
no tener derecho a gobernar. La novedad histórica de Spinoza reside, por tanto, en el modo en que desplaza el debate sobre el mejor gobierno. Ese debate no tiene que girar en torno a los marcos establecidos por la doctrina cristiana o por el pacto racional, que los gobernados, supuestamente, deben respetar, sino en el ejercicio actual del poder, en el modo en que el funcionamiento actual de la administración de los asuntos comunes aumenta o disminuye la potencia de la multitud y la de los ciudadanos.

Ahora bien, cambiar el objeto de debate significa cambiar el rango de los participantes en el mismo. El debate sobre la legitimidad cristiana o contractualista es un debate entre intérpretes, teólogos o filósofos, ya que el pacto se da siempre en la teoría (TTP 17: 201). El debate -o la disputa- sobre la gestión actual de la comunidad es un debate en la que no pueden dejar de participar todos los afectados por esa gestión. La teoría es inmediatamente práctica, ejercicio mismo de la política; y la práctica es inmediatamente teórica, pues no puede escapar de la puesta en común de los problemas de la vida en común.

Esto explica: 1. La diferencia entre el TTP y el TP. En el TTP, Spinoza excluye al vulgo del debate. 2. El papel de la multitud: ese debate ha empezado ya siempre, es la potencia misma de la multitud que actúa como una sola mente. 3. Los modelos de monarquía, aristocracia y democracia (faltante) suponen la construcción de las mejores condiciones para que esa disputa pueda producirse en beneficio de todos dentro de los parámetros que definen al poder supremo en cada régimen.

Los parágrafos que dan la clave para esta nueva perspectiva son, sin duda, TP 7/27 y TP 7/29. El primero puede leerse perfectamente como una autocrítica de Spinoza. La distinción entre filósofos y vulgo que marcaba el comienzo y todo el desarrollo del TTP queda en este parágrafo completamente superada. La arrogancia del poder, el causar miedo si no lo tienen, la falta de moderación o el quebranto de la verdad cuando no conviene a sus intereses no son patrimonio de los pobres y plebeyos, sino universales. Pero, lo que merece reseñarse para nuestro asunto es la respuesta de Spinoza a la acusación que los poderosos dirigen a la plebe de estar falta de verdad y de juicio.

Finalmente, que la plebe carezca de toda verdad y de juicio no es nada extraño cuando los principales asuntos del imperium se tratan a sus espaldas y ella no puede sino hacer conjeturas por los escasos datos que no se pueden ocultar. Porque suspender el juicio es una rara virtud. Pretender, pues, hacerlo todo a ocultas de los ciudadanos y que éstos no hagan juicios endebles ni lo interpreten todo torcidamente, es una necedad supina. 
Dicho de otro modo, es la exclusión de la plebe de la deliberación y la toma de decisiones respecto de los asuntos públicos, establecida por ciertas formas de organizar la sociedad, la que produce una plebe incapaz de participar en esos asuntos. No hay nada natural en la falta de verdad y juicio de la plebe, sino que ésta, si se da, es una consecuencia de la constitución política. Por tanto, siendo que los vicios son universales, la única manera de extender también las virtudes es modificando esa constitución para que se permita a la plebe dejar de ser tal y todas las personas puedan convertirse en actores políticos que participan en las decisiones que les afectan. He aquí el modo en que se concreta la utilidad común o concordia del mejor imperium, en que la potencia común aumenta gracias al aumento de la potencia de cada uno. La potencia es la capacidad para entrar en el debate en el que se define lo bueno y lo malo compartido

La posición de Spinoza es, por tanto, clara. La equiparación entre derecho y potencia, que a su vez supone la separación entre legitimidad y valoración, es la condición para que esa valoración deje de ser la función de unos intérpretes separados del resto de sus conciudadanos y se convierta en el objeto del debate de la multitud. Si en el TTP Spinoza piensa desde la distancia de los filósofos, en el TP se pone enteramente del lado de la multitud, de la multitud libre. Este es el modo en que la filosofía puede formar parte de la utilidad mutua, un uso mutuo del conocimiento de cómo vivir en común. Es el extremo opuesto a la desconfianza o el miedo mutuos de Hobbes ${ }^{18}$. Y se concreta en la deliberación común en la asamblea (concilium) o consejo, pieza central de los fundamentos del mejor imperium, sea monárquico, aristocrático o democrático. La asamblea es la forma de la cooperación intelectual que pone en práctica la unión de los ánimos para la conservación del mejor imperium. Y la multitud libre es la que se organiza a través de asambleas.

El mejor imperium es aquel con cuyos fundamentos la multitud organiza su cooperación intelectual, lo que Spinoza llama "concordia", simultánea de su cooperación material. Y el peor imperium es el que está privado de los fundamentos requeridos por una disputa liberadora, esto es, el fundado en el derecho de guerra. O dicho de otro modo, el mejor imperium es el que es deseado libremente, el que es deseado en la práctica de la libertad en la que consiste la participación en la definición compartida de lo bueno y lo malo, por la entera multitud, porque es ese deseo común el que lo califica de "mejor". Es el mejor modo de determinar en común qué es bueno y qué malo. No es el mejor, entonces, porque así lo dictamine un observador externo o neutral, interprete de Dios, de la Naturaleza, de la Razón o de la Multitud, sino en cuanto la multitud juzga un imperium como óptimo porque lo desea más que a otros. En su práctica filosófica en el TP, Spinoza no se entiende a sí mismo, entonces, como ese observador imparcial, sino como filósofo en la multitud.

\footnotetext{
${ }^{18}$ Hobbes, De cive, Prefacio, p. 8.
} 


\section{El deseo común}

La idea de una potencia común de la multitud (TP 3/9) implica necesariamente la de un deseo común de esa misma multitud. El miedo y la esperanza comunes, la indignación y el anhelo de venganza compartidos o el rechazo de la soledad de TP 3/9, TP 4/6 o TP 6/1, pero también la unión de los ánimos que hace posible la búsqueda por parte de la multitud de lo que es verdaderamente útil a todos en TP 3/7, nos remiten a un deseo común. Y nos muestran, además, que el deseo común puede ser pasional o racional.

Hemos visto que la indignación, ese odio compartido por identificación o amor a la víctima, lleva consigo la propia unión afectiva. El miedo común o el anhelo compartido de venganza, sin embargo, requieren un factor de unidad trascendente, una imagen de la destrucción de lo temido u odiado que produzca una alegría en la que encontrarse. Las teologías políticas habían presentado a Dios como poder de salvación eterna en cuya adoración imaginar el fin de todo lo detestable. El mecanismo de la representación que Hobbes expone en el Leviatán juega, también, en este terreno. El gobernante o asamblea que representa a los gobernados es, entre otras cosas, la imagen de la aniquilación de la discordia del estado de naturaleza superado, la garantía imaginaria de que no se va a volver al miedo y la miseria de la guerra de todos contra todos.

Spinoza no renuncia al uso de la esperanza como herramienta política, sobre todo como esperanza de gloria, de paz, de bienestar o de libertad, según expone, por ejemplo, en diferentes artículos de los capítulos VII y X del Tratado político. En TP 10/9 señala que "los derechos no pueden mantenerse incólumes, a menos que sean defendidos por la razón y por el común afecto de los hombres; de lo contrario, es decir, si sólo se apoyan en la ayuda de la razón, resultan ineficaces y fácilmente son vencidos". En TP 7/22 alude a la libertatis imago como incitamentum a la victoria del ejército popular. Pero, la producción social de las imágenes que excluyen lo temido u odiado no es, para él, lo que construye la comunidad en el mejor imperium, sino que se pone al servicio de la constitución del deseo común racional, de la cooperación material e intelectual de la multitud.

El representante hobbesiano es la imagen de la unidad, la persona artificial, que siendo una subsume a toda la multitud. Esta representación no sólo sienta las bases del Estado absoluto o separado hobbesiano, sino que atribuye al soberano la causa de la paz y el bienestar de todos, lo que, desde la perspectiva de Spinoza, si funciona, es de esperar que genere amor pasional o devoción de los súbditos hacia los mandatarios. Y, en consecuencia, se convertirá en un obstáculo para que los ciudadanos entiendan su propia participación en la configuración de la sociedad. 
La concordia, sin embargo, requiere un imperium no separado, absoluto precisamente en tanto que no separado de la multitud. Implica una interacción constructiva entre los individuos. Lo que no significa que no haya imperium o que desaparezca por entero el conflicto. La clave de la continuidad del proceso constructivo de concordia es que la potencia alcanzada con la cooperación tienda a redundar en el aumento de potencia de cada uno. Pero, ¿qué es en este caso lo que los une? La concurrencia, esto es, el hecho de producir un efecto conjunto, una potencia común, que persista y acreciente las fuerzas que lo generan.

La concurrencia hecha círculo virtuoso activa su propia afectividad. Es un ciclo de liberación política. El amor ya no es hacia una figura que representa el fin de la tristeza, sino hacia los diferentes individuos y colectivos que concurren y al proceso mismo, accidentado y contradictorio, que son la causa verdadera del aumento de potencia. El amor es a lo común que, articulando las diferencias, hace posible el encuentro sostenido de la multitud: un deseo común por lo común.

¿En qué coinciden la alegría compartida por imaginar la ausencia de lo que aborrecemos, incluso en su forma radical hobbesiana, y el deseo común por lo común de la concordia?

En ambas lógicas encontramos la actividad de un deseo resistente. Ahora bien, la alegría que nos produce imaginar la desaparición de lo odiado surge de un deseo resistente inconsciente de sí mismo. O, mejor, es consciente de sus efectos, pero inconsciente de sí mismo como causa. Es un deseo que se desconoce a sí mismo como causa porque, al ser motivado por una representación, no atiende a su colaboración en el aumento de potencia que vive como alegría.

La ilusión por ver desaparecer lo que tememos u odiamos es un caso de deseo resistente, pero un caso especial, porque tiende a reprimir el conocimiento del propio deseo. Es una forma de deseo resistente que reprime el conocimiento de sí. Este deseo resistente es, así, el fuera de campo $^{19}$ de la identidad comunitaria de la representación: aquello que, ocultándose, la constituye. Es una forma de deseo que reproduce la ignorancia, que dinamiza un ciclo político de servidumbre, que alimenta la impotencia de la multitud. $O$, más en concreto, el deseo es el fuera de campo en tanto que exclusión representada, ya que la representación está en lugar de lo excluido, como en Hobbes el soberano está en lugar de la multitud ${ }^{20}$. La potencia de la multitud de un régimen puramente representativo, como el imaginado por Hobbes, ocultaría su propia realidad. El deseo en la forma de la ignorancia de sí como causa es la condición de

\footnotetext{
${ }^{19}$ Para este uso de la metáfora del fuera de campo, cfr. Teresa de Lauretis, Technologies of Gender. Essays on Theory, Film, and Fiction, Hampshire, The Macmillan Press, 1989, p. 25.

${ }^{20}$ Hobbes, Leviatán, XVI.
} 
producción de la representación política, aquello sobre lo que ésta tiene que "mentir" para imponerse como absoluto ${ }^{21}$.

La filosofía política de Spinoza apunta a hacer consciente de sí ese deseo resistente incluso allí donde se niega a sí mismo como causa. Cuando Spinoza habla del mejor imperium o cuando se refiere al bien común o a la salvación o la salud común, entiende por tales expresiones no sólo aquello que la multitud desea y, porque lo desea, lo juzga como mejor o como bueno, sino a lo producido por la multitud en cuanto es consciente de su deseo como causa de las valoraciones compartidas y sabe de las valoraciones como efectos del deseo común. El mejor imperium es el instituido por una multitud que conoce su potencia como causa instituyente de lo bueno y lo malo compartido.

Dado, por otra parte, que el deseo de la multitud o deseo común es su misma esencia, no puede desear nada sin al mismo tiempo desear perseverar en el ser (E 4P21). El bien común es, por tanto, en primer lugar, la consciencia de la propia persistencia del deseo común. Lo que el deseo común juzga como bueno es su propia existencia y conservación. A esto se refiere Spinoza cuando señala en TP 1/6 que la virtud del imperium es la seguridad (securitas) o cuando en TP 5/2 afirma que el fin del estado civil es la paz y la seguridad de la vida (pax vitaque securitas). Se ha subrayado a menudo ${ }^{22}$ el contraste de estas fórmulas con el taxativo "Finis ergo reipublicae revera libertas est" de TTP 20: 227. Pero, el resto del capítulo V del TP, primero, y el conjunto de su obra política, después, parece confirmar que el contraste es más una cuestión de énfasis. Spinoza no separa seguridad y libertad, por la misma razón que no separa potencia (o virtud) y libertad en la Ética. La seguridad no parece ser algo distinto de la potencia o conatus de la sociedad o la intensidad del deseo común. En el mismo capítulo V del Tratado político, lo que se opone a la seguridad es o bien la dominación (TP 5/4-6), o bien la imprudencia de la multitud (TP 5/7). El bien común es, por tanto, inmanente en Spinoza en la medida en que es el valor de la propia perseverancia de la libertad común (TP 10/8).

El deseo común, por lo demás, es un deseo político, algo que, como hemos visto, implica que asume la irreductibilidad del conflicto. Y, en consecuencia, es un deseo que surge también de afectos comunes que son pasiones. En Spinoza, esto significa, por de pronto, que los afectos conflictivos son o pueden ser contenidos o limitados con otros afectos comunes. Y que el deseo común está tramado sobre la imitación de los afectos y sus efectos socialmente reguladores hacia fuera y hacia dentro.

${ }^{21}$ Hablar de un deseo que se desconoce como causa es otra forma de expresar la ilusión de la voluntad libre, que en este caso actúa a nivel social. Es la servidumbre voluntaria como servidumbre en la ilusión de la voluntad libre. Cfr. Stefano Visentin, "Volonté d'être esclave et désir d'être libre. Ambivalence de la multitude chez Spinoza", [en Chantal Jaquet y Pierre-François Moreau (dirs.), Spinoza transalpin: Les interprétations actuelles en Italie, París, Éditions de la Sorbonne, 2012], pp. 190 y ss.

${ }^{22}$ Cfr. por ejemplo, Étienne Balibar, Spinoza et la politique, op. cit., pp. 63-64. 
Pero, quizás, la cuestión más importante es si lo que predomina en el deseo común es el determinante pasional o el racional. Por un lado, Spinoza pone a las pasiones o "la condición o naturaleza común de los hombres" (TP 1/7) como fundamento del deseo común. Insiste tanto en el TTP como en el TP en que los bárbaros también viven en sociedad ${ }^{23}$. O habla, en TP $6 / 1$, de una conveniencia natural por la que la multitud busca conducirse como una sola mente no por la razón, sino por afectos comunes que son pasiones ${ }^{24}$. Esta base social pasional es una expresión del principio de relacionalidad múltiple al nivel de la sociedad humana. La relacionalidad esencial de todos los modos de la sustancia se expresa en los modos humanos como socialidad $\mathrm{y}$, más en concreto, como imitación de los afectos y emulación. No hay, por tanto, origen (transcendente) de la sociedad por la misma razón que no hay origen (transcendente) de la humanidad. La relacionalidad y el conatus son dos principios que se coimplican, que son igualmente fundamentales, y que hay que entender siempre en su singularidad, en este caso, en el proceso de producción y reproducción de la sociedad humana.

Ahora bien, el deseo verdaderamente común es el guiado por la razón. Es el deseo generado por el conocimiento de la verdadera utilidad. Es el deseo común autónomo del que trata el capítulo V del TP. Y esto por dos motivos. Por un lado, porque la conveniencia necesaria entre los hombres se da a través de la razón, como explica Spinoza en E 4P35. Y por otro, porque para alcanzar la seguridad que proporciona la libertad, hay que evitar las pasiones que sostengan ideas transcendentes. El afecto común no puede ser una alegría acompañada por la imagen de un salvador, ya sea un supuesto "poseedor de la luz divina" (TTP P: 8), o un representante de la multitud, en el sentido hobbesiano. El afecto común no puede ser la admiración hacia unos u otros ambiciosos que utilizan la superstición o el miedo para dominar sobre los corazones de los seres humanos y para convertirlos de seres racionales en brutos ${ }^{25} \mathrm{o}$ autómatas (TTP 20: 241). La seguridad, la potencia, que proporciona la libertad sólo puede lograrse si los afectos comunes son aquellos en los que puede crecer un deseo común racional; si son los afectos de una multitud que se conduce más por la esperanza que por el miedo, que cultiva la vida, en lugar de huir de la muerte, que vive para sí y no para quien la ha derrotado; si, en definitiva, son los afectos de una multitud libre y no los de una multitud sierva (TP 5/6).

${ }^{23}$ Cfr. TTP 5: 59 y TP 1/7.

24 Esto es, que no nacen de la razón (“quae ex ratione non oriuntur"), como señala en TP 2/5. Aunque en el TP la diferencia entre afectos y pasiones vacila y más bien predomina la oposición entre razón y afectos comunes como en TP 6/1 o en TP 10/9. Ni en esta obra ni en ninguna otra usa Spinoza la expresión "pasiones comunes".

25 "quae homines ex rationalibus brutos reddunt" (TTP P: 8).

Araucaria. Revista Iberoamericana de Filosofia, Política, Humanidades y Relaciones Internacionales, año $21, \mathrm{n}^{\circ} 42$. Segundo semestre de 2019. Pp. 23-44. ISSN 1575-6823 e-ISSN 2340-2199 doi: 10.12795/araucaria.2019.i42.02 


\section{Conclusión}

No se trata, pues, de desechar las pasiones, sino de entablar una batalla en su seno mismo, en el modo como entendemos los propios juicios de valor, ya que las valoraciones son la consciencia de los afectos (E 4P8). En la propia concepción de los valores se juega la posibilidad de lo transcendente o de lo inmanente. Una concepción transcendente de las valoraciones sostiene una admiración común hacia una fuente de salvación, sea Dios hecho Iglesia o sea el pacto originario hecho Estado, que se percibe como separada, como autónoma con respecto a la constitución efectiva del deseo común. Y el espejismo de separación es provocado precisamente porque la fuente de salvación es concebida también como fuente de los valores. Dios o el pacto originario separan el bien del mal. Los seres humanos desconocen su actividad como productores de los valores al tiempo que se la atribuyen a una realidad que se sitúa fuera de su alcance y ante la cual sólo saben comportarse sumisamente $(\mathrm{E} 1 \mathrm{Ap})^{26}$.

El conocimiento por parte de la multitud de su propia potencia como causa instituyente, la seguridad entendida como potencia, la irreductibilidad del conflicto y el deseo común racional confluyen. De ahí la importancia política de la ontología de Spinoza. De ahí lo esencial de negar la posibilidad de una normatividad metafísicamente fundada en un origen transcendente, sea Dios o el Pacto. Toda normatividad racional, ética o política, es fruto de los deseos singulares y comunes de los seres humanos históricamente activos, también el bien común o el mejor imperium, ya que su razón de existir es el conatus humano o el conatus de la multitud como conatus naturales entre otros infinitos conatus naturales y en un universo natural sin centro ni jerarquías ontológicas, sin ejes de referencia ni lugares privilegiados ${ }^{27}$. La normatividad racional es relativa a nuestro conatus pero no indiferente. Descansa en el conocimiento de aquello que nos es útil, sobre todo en el conocimiento de que lo más útil para el hombre es el hombre. Pero, también en el conocimiento de nuestra condición o naturaleza común, esto es, en el hecho de que somos parte de la naturaleza y estamos sometidos a las pasiones. La normatividad racional tiene que buscar en cada coyuntura la mejor forma de contener las pasiones y ampliar

\footnotetext{
${ }^{26}$ Es un mecanismo similar a la alienación política que luego encontrará el Marx más cercano a Feuerbach en la filosofía política de Hegel. Cfr. Karl Marx, Crítica de la Filosofía del Estado de Hegel, Madrid, Biblioteca Nueva, 2002, pp. 97 y ss.

27 María Luisa de la Cámara ha explicado esta característica de la filosofía de Spinoza como "un eclipse de la autoridad" tradicional, que se expresa de manera rotunda en la Ética: "la nueva doctrina no propone la trascendencia de ninguna estructura metafísica, con lo que desaparece también toda forma de jerarquía metafísica. Nada es superior y/o mejor que nada: ni Dios sobre la naturaleza, ni el alma sobre el cuerpo, ni el intelecto sobre los afectos. Ni un hombre sobre otro". Cfr. María Luisa de la Cámara, "La teoría de Spinoza sobre la autoridad", en Araucaria. Revista Iberoamericana de Filosofia, Política y Humanidades, año 20, no 39. Primer semestre de 2018, p. 242.
} 
nuestro conocimiento sobre lo útil. En ambas tareas se las tiene que ver con las normatividades existentes, esto es, con las ideas universales de la naturaleza humana de carácter transcendente o escéptico vigentes en un momento histórico determinado, y con sus respectivas definiciones del mal. Y tiene que afrontar los mecanismos de la imitación de los afectos que funcionan a partir de esas ideas normativas de humanidad y de maldad y que sólo son modificables en la medida en que se conoce su dinámica reguladora. Por todo ello, el pensador político inmanentista tiene que operar siempre ya en un espacio de construcción social de los valores en el que no puede actuar si no lo hace en cooperación con otras personas. Y su razón es una razón polémica, en disputa, también consigo misma, no un remanso de imparcialidad.

El deseo verdaderamente común, el deseo racional colectivo sólo puede ser generado por la propia vida en común de la multitud y en la disputa por las definiciones compartidas de lo bueno y lo malo, en la disputa en torno a la institución del imperium. El deseo común supone la cooperación intelectual de la multitud, sin exclusiones ${ }^{28}$, que define el bien común como consciencia de su deseo. Los modelos de mejor imperium que Spinoza empieza a inventar a partir del capítulo VI del TP son diseños de esa cooperación mental y material formadora de deseo común. Su aportación a un proceso cooperativo que necesariamente va mucho más allá de su obra y llega, como sin darnos cuenta, hasta nosotros.

El devenir multitud del filósofo inmanentista fluye en el devenir inmanente, libre, de la multitud. Sólo en la medida en que el deseo común proviene de una alegría compartida puede producir bien común. El mejor imperium es aquel donde prospera la alegría, la vida racional y el amor. El instituido por la multitud libre. Gobernar bien no es lo mismo que tener derecho a gobernar. Como dice Spinoza al final de TP 5/6, en nada se diferencian los derechos respectivos del imperium instituido por la multitud libre y del sostenido en la dominación de unos sobre otros. La oposición entre ellos no se halla en sus derechos, sino en sus medios y sus fines. Mientras se gobierna, se gobierna siempre con derecho, aunque sea acumulando muerte, miedo y tristeza (TTP 20: 240). La clave no está, pues, en gobernar sin más, sino en hacerlo bien: en avivar la llama de la multitud alegre, potente, libre. En fundar el autogobierno de la multitud.

\footnotetext{
28 "Integra multitudo" en el latín de Spinoza. Aquí falta, por supuesto, identificar el desajuste, que se hace más evidente a partir del capítulo VI del Tratado político, entre la multitud entera y las exclusiones que el propio Spinoza da por sentadas o, incluso, justifica.
} 


\section{Referencias bibliográficas:}

\section{De Spinoza}

Spinoza, Baruch de, Opera, (4 vols.), ed. de C. Gebhardt, Heidelberg, Carl Winters, 1972 ( $1^{\text {a }}$ edición, 1925).

—, Ética, trad. Vidal Peña, Madrid, Editora Nacional, 1980.

—, Ética, trad. Atilano Domínguez, Madrid, Trotta, 2000.

-, Tratado teológico-político, trad. Atilano Domínguez, Madrid, Alianza, 1986.

-, E Evres III, Tractatus theologico-politicus / Traité théologique-politique, texto Fokke Akkerman, trad. Jaqueline Lagrée y Pierre François Moreau, París, PUF, 1999.

—, Tratado político, trad. Atilano Domínguez, Madrid, Alianza, 1986.

—, Traité politique, trad. Bernard Pautrat, París, Allia, 2013.

-, Euvres V, Tractatus politicus / Traité politique, texto Omero Proietti, trad. Charles Ramond, París, PUF, 2005.

—, Correspondencia, trad. Atilano Domínguez, Madrid, Alianza, 1988.

- Correspondencia completa, trad. Juan Domingo Sánchez Estop, Madrid, Hiperion, 1988.

\section{Otras obras citadas}

Aquino, Tomás de, La monarquía, Madrid, Tecnos, 1989.

—, Suma de Teología IV, Madrid, BAC, 1994.

Balibar, Étienne, Spinoza et la politique, París, P.U.F., 1985.

—, Spinoza politique. Le transindividuel, París, PUF, 2018.

Cámara, María Luisa de la, "La teoría de Spinoza sobre la autoridad", en Araucaria. Revista Iberoamericana de Filosofía, Política y Humanidades, año 20, no 39. Primer semestre de 2018.

Colom González, Francisco, "Legitimidad política" [en Fernando Quesada (ed.), Filosofía política I. Ideas políticas y movimientos sociales, Madrid, Trotta, 2002], pp. 171-186.

Espinosa, Francisco Javier, "Être une multitude et agir comme une seule âme", [en Maria Luísa Ribeiro Ferreira, Diogo Pires Aurélio, Oliver Feron (eds.), Spinoza, ser e agir, Lisboa, CFUL, 2011], pp. 129-151.

Hobbes, Thomas, Leviathan, Oxford, OUP, 1996.

- Tratado sobre el ciudadano, Madrid, Trotta, 1999.

Jaquet, Chantal, Les expressions de la puissance d'agir chez Spinoza, París, Éditions de la Sorbone, 2005.

Lauretis, Teresa de, Technologies of Gender. Essays on Theory, Film, and Fiction, Hampshire, The Macmillan Press, 1989. 
Matheron, Alexander, Études sur Spinoza et les philosophies de l'âge classique, Lyon, ENS Éditions, 2011.

Marx, Karl, Crítica de la Filosofía del Estado de Hegel, Madrid, Biblioteca Nueva, 2002.

Morfino, Vittorio, El tiempo de la multitud, Ciempozuelos, Tierradenadie ediciones, 2014.

Sainz Pezonaga, Aurelio, "Universalismo y singularidad en la Ética de Spinoza", [en Montserrat Galcerán Huguet y Mario Espinoza Pino, Spinoza contemporáneo, Ciempozuelos, Tierradenadie ediciones, 2009], pp. 133-160.

Tosel, André, Spinoza ou l'autre (in)finitude, París, L'Harmattan, 2008.

Zourabichvili, François, Spinoza. Une physique de la pensée, París, PUF, 2002. 